\title{
Compendio y Análisis de Medidas de Evaluación de la Inteligencia Emocional Capacidad
}

\author{
Compendium and Analysis of Measures of Ability Emotional Intelligence
}

\author{
Débora Rodrigo-Ruiz ${ }^{1}$, Javier Cejudo ${ }^{2}$ y Juan Carlos Pérez-González ${ }^{3}$
}

\begin{abstract}
Resumen
Debido al interés que la literatura científica ha mostrado en la inteligencia emocional y los beneficios que ésta parece aportar sobre diferentes áreas de la persona, el presente trabajo realiza una recopilación y análisis de los diferentes instrumentos de evaluación de la inteligencia emocional capacidad hallados tras una revisión selectiva de la literatura. Se analizan las propiedades psicométricas y las características distintivas de cada uno de los instrumentos localizados teniendo en cuenta su adecuación a la medición del constructo. Se concluye que, a pesar de la aparente diversidad de pruebas, el desarrollo de instrumentos de evaluación de la inteligencia emocional capacidad con garantías psicométricas se encuentra aún en un periodo inicial.
\end{abstract}

Palabras clave: evaluación, diagnóstico, inteligencia emocional, test, validez de constructo

\begin{abstract}
Due to the interest that the scientific literature has shown in emotional intelligence and the benefits that it seems to produce in different vital areas, this work compiles and analyses the different instruments for measuring the ability emotional intelligence found after a selective review of the literature. The psychometric properties and the distinctive features of each one of the found instruments are analysed taking into account their adequacy to the assessment of the construct. It is concluded that, despite the apparent diversity of instruments, the development of ability emotional intelligence assessment instruments with psychometric guarantees is still in an initial period.
\end{abstract}

Keywords: assessment, diagnosis, emotional intelligence, test, construct validity

\footnotetext{
${ }^{1}$ Doctora en Educación con Premio Extraordinario de Doctorado (PED). Licenciada en Psicopedagogía. Profesora colaboradora de EDUEMO, Laboratorio de Educación Emocional de la Universidad Nacional de Educación a Distancia (UNED). C/Juan del Rosal, 14, 28040 Madrid, España. Correo: deborarodrigo@gmail.com

${ }^{2}$ Doctor en Educación con PED. Licenciado en Psicología y en Psicopedagogía. Profesor contratado doctor del Departamento de Psicología de la Universidad de Castilla-La Mancha. Ronda de Calatrava, 3, 13071 Ciudad Real, España. Correo: manueljavier.cejudo@uclm.es

${ }^{3}$ Doctor Europeo en Educación con PED. Licenciado en Psicopedagogía. Profesor Titular de Métodos de Investigación y Diagnóstico en Educación (MIDE) de la Facultad de Educación de la UNED. C/Juan del Rosal, 14, 28040 Madrid, España. Correo: jcperez@edu.uned.es
} 


\section{Introducción}

La inteligencia emocional (en adelante IE) alude a diferencias individuales en la percepción, expresión, comprensión y regulación de estados emocionales, emociones y sentimientos en uno mismo y en los demás (Nelis, Quoidbach, Mikolajczak, \& Hansenne, 2009), y constituye un constructo de enorme relevancia en psicología y en educación (Bisquerra, Pérez-González, \& García Navarro, 2015; Pérez-González \& Qualter, 2018; Qualter et al., 2017), siendo específicamente la cuestión de su evaluación y diagnóstico un tema de permanente atención en la literatura (e.g., Martorell, 2005; Siegling, Saklofske, \& Petrides, 2015).

La extensa investigación empírica ha demostrado que esta característica psicológica es beneficiosa para una variada diversidad de funciones vitales, con efectos positivos sobre variables como el desempeño académico, social y laboral, y especialmente sobre el bienestar y la salud (e.g., Fernández-Berrocal \& Extremera, 2016; Petrides et al., 2016).

Asimismo, recientemente se ha demostrado que la IE se puede mejorar a través de apropiadas intervenciones educativas (Hodzic et al., 2017). Sin embargo, la mayor parte de las intervenciones analizadas en este meta-análisis están dirigidas a mejorar la IE como rasgo de personalidad (IE rasgo), pero no como IE capacidad, es decir, no como una capacidad para razonar sobre las emociones (Mayer, Caruso, \& Salovey, 2016). De modo que el meta-análisis de Hodzic et al. (2017) es principalmente una evidencia de que los programas de entrenamiento de la IE rasgo son eficaces, pero apenas constituye una prueba sólida en favor de la eficacia de programas de entrenamiento de la IE capacidad, debido al reducido número de investigaciones disponibles hasta el momento que se hayan centrado en mejorar la IE capacidad. Esto implica que es necesaria aún mucha más investigación evaluativa de programas de intervención dirigidos a mejorar la IE capacidad o sus componentes. Este tipo de investigación pasará, necesariamente, por una apropiada selección de pruebas de evaluación fiables y válidas que garanticen una buena recogida de información en las medidas pre y post de evaluación de la eficacia de los programas. No obstante, la literatura al respecto informa de la dificultad de encontrar instrumentos para la medida de capacidades emocionales, especialmente en nuestro idioma (e.g., Guaita, 2007).

Con objeto de facilitar esta labor a investigadores y educadores surge el trabajo que aquí presentamos.

Uno de los mayores obstáculos que ha sufrido la IE a lo largo de estos años de investigación, ha sido la confusión entre los constructos IE capacidad e IE rasgo y el uso indistinto de diferentes tipos de instrumentos para evaluar tanto uno como otro constructo. Es de suma importancia recordar que se trata de dos constructos bien diferenciados y que, por lo tanto, deben ser evaluados con instrumentos de diferentes características (Pérez-González, Petrides, \& Furnham, 2007; Siegling et al., 2015).

Los instrumentos de IE rasgo deberían ser capaces de medir características o rasgos de la personalidad que provocan en el individuo una tendencia a actuar de una determinada manera. Lo más acertado en este caso es preguntarle al individuo cuál sería su comportamiento en determinado caso y que fuera él mismo quien nos diera esa información. Este tipo de medidas, utilizadas durante años para la medida de rasgos de personalidad, son consideradas en psicología y educación como medidas de rendimiento típico (Cronbach, 1949), en las que no existe una única respuesta correcta.

Sin embargo, no es adecuado utilizar medidas de rendimiento típico para la evaluación de la IE capacidad. La IE capacidad se refiere a un conjunto de capacidades de procesamiento de información. Estamos por tanto ante una presunta capacidad cognitiva. En este caso, sería viable pedir a un sujeto que nos diera información acerca de su propio intelecto a través de una medida de rendimiento típico, pero dicha información debería ser interpretada con mucha precaución (Schulze, Wilhelm, \& Kyllonen, 2007), puesto que para poder informar de un resultado, el sujeto debería haber realizado primero una prueba objetiva que le dé una puntuación sobre la que informar; en caso contrario, debería optarse por la estimación absoluta de dicha puntuación, lo que nos demuestra la insuficiencia de las medidas de rendimiento típico en la evaluación de una capacidad 


\section{REGULACIÓN REFLEXIVA DE LAS EMOCIONES PARA PROMOVER EL CRECIMIENTO EMOCIONAL}

E INTELECTUAL (R)

4.6. Gestionar eficazmente las emociones de los demás para lograr un resultado deseado

4.5. Gestionar eficazmente las emociones propias para lograr un resultado deseado (e.g., moderando las emociones negativas y realzando las positivas, sin reprimir o exagerar la información que éstas conllevan)

4.4. Evaluar estrategias para mantener, reducir o intensificar una respuesta emocional

4.3. Supervisar las reacciones emocionales (con relación a uno/a mismo/a y a los demás) para determinar, cómo de claras, típicas, influyentes o razonables son

4.2. Aferrarse a una emoción o bien desligarse de ella en función del juicio hecho acerca de su utilidad o de su valor informativo

4.1. Permanecer abierto/a a los sentimientos, tanto a los agradables como a los desagradables, según sea necesario, y a la información que transmiten

\section{COMPRENSIÓN Y ANÁLISIS DE LAS EMOCIONES; EMPLEO DEL CONOCIMIENTO EMOCIONAL (U)}

3.8. Reconocer diferencias culturales en la evaluación de las emociones

3.7. Comprender cómo una persona podría sentirse en el futuro o bajo ciertas condiciones (previsión afectiva)

3.6. Reconocer las transiciones probables entre las emociones (e.g., como la transición de la ira a la satisfacción, o de la ira a la culpabilidad o la vergüenza)

3.5. Comprender sentimientos complejos y mezclados (e.g., sentimientos simultáneos de amor y odio, o mezclas tales como el "pavor", como una combinación de miedo y de sorpresa)

3.4. Diferenciar entre estados de ánimo y emociones

3.3. Valorar las situaciones probables de elicitar distintas emociones

3.2. Determinar los antecedentes, significados y consecuencias de las emociones (e.g., la tristeza a menudo viene acompañada de una pérdida)

3.1. Etiquetar las emociones y reconocer las relaciones entre ellas (e.g., entre las palabras y las emociones, como la relación entre gustar y amar)

\section{FACILITACIÓN EMOCIONAL DEL PENSAMIENTO (F)}

2.5. Seleccionar los problemas en función de cómo el estado emocional en curso podría facilitar la cognición

2.4. Aprovechar los cambios de humor para generar diferentes perspectivas cognitivas (e.g., de optimista a pesimista, estimulando la consideración de múltiples puntos de vista)

2.3. Priorizar el pensamiento dirigiendo la atención (hacia la información importante) congruente con el sentimiento presente

2.2. Generar emociones como un medio para conectar con las experiencias de otra persona

2.1. Generar emociones como una ayuda para el juicio y la memoria (en relación a los sentimientos)

\section{PERCEPCIÓN, VALORACIÓN, Y EXPRESIÓN DE LAS EMOCIONES (PE)}

1.7. Identificar expresiones emocionales engañosas o falsas.

1.6. Discriminar entre expresiones emocionales precisas vs. imprecisas

1.5. Comprender cómo las emociones son mostradas dependiendo del contexto y la cultura

1.4. Expresar las emociones con exactitud y expresar las necesidades relacionadas con esos sentimientos

1.3. Percibir contenido emocional en el entorno, las artes visuales y la música

1.2. Percibir emociones en otras personas a través de sus señales vocales, expresión facial, lenguaje y comportamiento

1.1. Identificar emociones en los estados físicos, sentimientos, y pensamientos de uno/a mismo/a

Figura 1. Modelo de IE capacidad actualizado de Mayer et al. (2016)

(Pérez-González et al., 2007; Siegling et al., 2015).

En concreto, para la medida de la IE capacidad, lo que se requiere es una prueba que discrimine entre respuestas correctas e incorrectas $\mathrm{y}$ en las que puedan establecerse objetivamente los aciertos y errores que nos permitirán determinar si se dispone o no de dicha capacidad o en qué grado. Para ello se emplean medidas de rendimiento máximo, en las que el sujeto debe afrontar una tarea que requerirá la puesta en práctica de las capacidades que desean medirse, y así, en función del desempeño que se realice, podremos hablar de una mayor o menor capacidad. Este es el tipo de pruebas habituales para realizar la evaluación cognitiva y de diferentes aptitudes intelectuales (e.g., Cronbach, 1949).

En cuanto al dominio muestral de la IE capacidad, hasta la fecha el modelo teórico que lo ha definido de forma más detallada es el de Mayer y Salovey (1997), recientemente revisado y extendido por los mismos autores (i.e., Mayer et al., 2016). Una síntesis del dominio muestral de la IE capacidad definido por este modelo se ofrece en la Figura 1. Este modelo de la IE capacidad ha 
sido denominado por Pérez-González (2010) como el modelo PEFUR, acrónimo formado por los términos en inglés de las áreas o ramas emocionales que según Mayer y Salovey (1997) constituyen la IE capacidad ( $P$ : perception; $E$ : expression; F: facilitation; $U$ : understanding; $R$ : regulation). Aunque según este modelo teórico la IE capacidad se divide en cuatro componentes o ramas, de acuerdo con Izard, Trentacosta, King, Morgan y Diaz (2007) la rama facilitación emocional del pensamiento (también denominada de utilización emocional) es en verdad parte de la regulación emocional. Asimismo, la investigación factorial y psicométrica de la IE capacidad omite la rama de facilitación (F) como parte del constructo (MacCann, Joseph, Newman, \& Roberts, 2014). Por tanto, si bien el modelo teórico de la IE capacidad sugiere cuatro componentes, parece más sólido considerar que la IE capacidad se basa en únicamente tres subcapacidades o factores "empíricos" (i.e., PEUR): percepción/expresión emocional, comprensión emocional, y regulación emocional.

Dada la confusión presentada entre instrumentos de IE capacidad e IE rasgo, y la importancia de disponer de instrumentos válidos y fiables para la medida de la IE capacidad en la implementación de programas de mejora de la IE, se presenta este trabajo destinado a la identificación de posibles instrumentos de IE capacidad. Identificar y compilar estos instrumentos, presentes en la literatura científica, permitirá su uso en la evaluación de programas de intervención en educación emocional, aumentando la precisión de los mismos.

\section{Método}

En el presente trabajo se realiza una revisión selectiva no sistemática de la literatura con el objetivo de identificar el mayor número de instrumentos existentes en la actualidad para la medida de la IE capacidad. Las fuentes a tomar en cuenta serán en exclusiva fuentes publicadas por revistas académicas y/o de editoriales de prestigio. Se comenzará con una búsqueda de instrumentos de IE capacidad sobre los que se determinarán las facetas medidas por cada uno y se analizará su conveniencia según cada uno de los factores empíricos de la IE capacidad (Mayer et al., 2016; MacCann et al., 2014).

Mayer, Roberts y Barsade (2008) realizan una clasificación de las medidas según su diseño y estructura clasificándolas en medidas basadas en capacidades específicas, refiriéndose a aquellas que realizan la medición sobre algunas de las facetas de la IE capacidad, y medidas basadas en modelos integradores, que involucran las capacidades mentales de múltiples áreas de la IE. En este trabajo, y a la luz de la literatura precedente (Bisquerra et al, 2015), diferenciaremos dos tipos de medidas de la IE capacidad según su grado de representatividad del dominio muestral del constructo. De este modo, haremos distinción entre medidas comprehensivas, aquellas que realizan una evaluación sobre todas o la mayoría de las ramas y facetas que componen el dominio muestral de la IE capacidad; y medidas parciales, aquellas cuya medición se produce sobre únicamente alguna o algunas de las ramas o facetas.

Cabe precisar que esta revisión se limitará exclusivamente a pruebas estandarizadas objetivas tipo test.

\section{Resultados}

Tras una búsqueda de los diferentes instrumentos de IE recogidos en la literatura científica, hemos identificado un total de 34 instrumentos de medida de la IE capacidad global o de algunos de sus componentes.

A continuación, realizamos un análisis de los diferentes instrumentos observando sobre qué elementos realizan su medición y cuáles son los indicadores psicométricos con referencia a la fiabilidad y validez de cada uno de ellos. En primer lugar, nos centraremos en el contenido de las diferentes pruebas atendiendo a las capacidades pertenecientes a cada una de las ramas o factores sobre las que se realiza la medición. Como puede observarse en la Tabla 1, los datos presentados reflejan ya una información detallada sobre la naturaleza de las pruebas, que pasaremos a analizar a continuación, pero en la que observamos a primera vista una destacada primacía de la evaluación realizada sobre las capacidades pertenecientes a la primera rama del 
Tabla 1. Instrumentos para la medida de la IE capacidad y ramas que miden del Modelo de Mayer y Salovey (1997)

\begin{tabular}{|c|c|c|c|c|}
\hline \multirow{2}{*}{ Instrumento } & \multicolumn{4}{|c|}{ Rama medida } \\
\hline & $\mathrm{R} 1$ & $\mathrm{R} 2$ & R3 & R4 \\
\hline $\begin{array}{l}\text { CARAT. Communication of Affect Receiving Ability Test (Buck, 1976; CARAT-SPR: } \\
\text { Buck, Powers, y Hull, 2017) }\end{array}$ & $\checkmark$ & & & \\
\hline PONS. Profile of Nonverbal Sensitivity (Rosenthal et al., 1979) & $\checkmark$ & & & \\
\hline CFT. Chimeric Faces Test (Levine y Levy, 1986) & $\checkmark$ & & & \\
\hline IPT. Interpersonal Perception Task (Costanzo y Archer, 1989) & $\checkmark$ & & & \\
\hline LEAS. Levels of Emotional Awareness Scale (Lane et al., 1990) & & & $\checkmark$ & \\
\hline MTAP. Minnesota Test of Affective Processing (Lai et al., 1991) & $\checkmark$ & & & \\
\hline $\begin{array}{l}\text { DANVA2-AF. Diagnostic Assessment of Non Verbal Affect-Adult Facial Expressions } \\
\text { (Nowicki y Carton, 1993) }\end{array}$ & $\checkmark$ & & & \\
\hline $\begin{array}{l}\text { CASP. The Child and Adolescent Social Perception Measure (Magill-Evans et al., } \\
\text { 1995) }\end{array}$ & $\checkmark$ & & & \\
\hline $\begin{array}{l}\text { DANVA2-AP. Diagnostic Assessment of Non Verbal Affect-Adult Paralanguage } \\
\text { (Baum y Nowicki, 1998) }\end{array}$ & $\checkmark$ & & & \\
\hline FAB. Florida Affect Battery (Bowers et al., 1999) & $\checkmark$ & & & \\
\hline MEIS. Multi-factor Emotional Intelligence Scale (Mayer et al., 1999) & $\checkmark$ & $\checkmark$ & $\checkmark$ & $\checkmark$ \\
\hline $\begin{array}{l}\text { JACBART. Japanese and Caucasian Brief Affect Recognition Test (Matsumoto et al., } \\
\text { 2000) }\end{array}$ & $\checkmark$ & & & \\
\hline RAFL. Recognition of Affect in a Foreing Language (Scherer, inédito) & $\checkmark$ & & & \\
\hline ERT. Emotion Recognition Test (Shimokawa et al., 2000) & $\checkmark$ & & & \\
\hline RMET. Reading the Mind in the Eyes Test (Baron-Cohen et al., 2001) & $\checkmark$ & & & \\
\hline EARS. Emotional Accuracy Research Scales (Geher et al., 2001) & $\checkmark$ & & & \\
\hline Emotional Stroop (Sharma y McKenna, 2001) & $\checkmark$ & & & \\
\hline FEFA. Frankfurt Test and Training of Social Affect (Bölte et al., 2002) & $\checkmark$ & & & \\
\hline MSCEIT. Mayer-Salovey-Caruso Emotional Intelligence Test (Mayer et al., 2002) & $\checkmark$ & $\checkmark$ & $\checkmark$ & $\checkmark$ \\
\hline EMT. Emotion Matching Task (Izard et al., 2003) & $\checkmark$ & & & \\
\hline ERT. Emotional Recognition Test-Revised (Lee et al., 2004) & $\checkmark$ & & & \\
\hline $\begin{array}{l}\text { DANVA2-POS. Diagnostic Analysis of Nonverbal Acuracy } 2 \text { Posture Test (Pitterman } \\
\text { y Nowiki, 2004) }\end{array}$ & $\checkmark$ & & & \\
\hline EIT. Emotional Inspection Time (Austin, 2005) & $\checkmark$ & & & \\
\hline $\begin{array}{l}\text { MP-EMA. Maximum-Performance Emotional Management Abilities (Freudenthaler y } \\
\text { Neubauer, 2005) }\end{array}$ & & & & $\checkmark$ \\
\hline Emotion differentiation card-sorting task (Kang et al., 2005) & & & $\checkmark$ & \\
\hline CATS. Comprehensive Affect Testing System (Froming et al., 2006) & $\checkmark$ & & & \\
\hline STEU. Situational Test of Understanding (MacCann y Roberts, 2008) & & & $\checkmark$ & \\
\hline STEM. Situational Test of Emotion Management (MacCann y Roberts, 2008) & & & & $\checkmark$ \\
\hline The NimStim set of facial expresions (Tottenham et al., 2009) & $\checkmark$ & & & \\
\hline MERT. Multimodal Emotion Recognition Test (Bänziger et al., 2009) & $\checkmark$ & & & \\
\hline ERI. Emotion Recognition Index (Scherer y Scherer, 2011) & $\checkmark$ & & & \\
\hline $\begin{array}{l}\text { STJ-based measure of EI. Situational Judgment Test-based measure of Emotional } \\
\text { Intelligence (Sharma et al., 2013) }\end{array}$ & $\checkmark$ & & $\checkmark$ & \\
\hline $\begin{array}{l}\text { TIEFBA. Test de Inteligencia Emocional de la Fundación Botín (Fernández-Berrocal } \\
\text { et al., 2018) }\end{array}$ & $\checkmark$ & $\checkmark$ & $\checkmark$ & $\checkmark$ \\
\hline
\end{tabular}

Nota. R1: rama 1; percepción y expresión emocional; R2: rama 2; facilitación emocional; R3: rama 3; comprensión emocional; R4: rama 4; regulación emocional.

modelo: la percepción y expresión de emociones. Además, es notorio que sólo tres instrumentos realizan una medición comprehensiva de todas o la mayoría de las capacidades de la IE capacidad. Asimismo, destacamos la poca representatividad entre los instrumentos revisados que tienen las últimas ramas de la IE capacidad, especialmente la cuarta: regulación emocional.

A continuación, en la Tabla 2 se presentan las propiedades psicométricas descritas en la literatura (e.g., Pérez-González et al., 2007; Fernández-Berrocal, Ruiz-Aranda, Salguero, Palomera,
\& Extremera, 2018) para las medidas comprehensivas de IE capacidad existentes junto con otras de sus características. Se muestran los datos referentes a la segunda versión del MSCEIT (Mayer, Salovey, \& Caruso, 2002b), la más actualizada de la que se dispone en los momentos en que se realiza el presente trabajo, que son considerablemente mejores que los obtenidos en una versión anterior del mismo (.68-.71; Mayer, Salovey, \& Caruso, 2002a). En la Tabla 3 pueden observarse las principales características de los instrumentos parciales para la medida de la faceta 
Tabla 2. Instrumentos comprehensivos de evaluación de la IE capacidad y sus características. (Adaptado de Pérez-González et al., 2007; Fernández-Berrocal et al., 2018)

\begin{tabular}{|c|c|c|c|c|}
\hline Instrumento & Fiabilidad & Validez & $\begin{array}{l}\text { Sistema de } \\
\text { puntuación }\end{array}$ & $\begin{array}{c}\text { Versión en } \\
\text { español }\end{array}$ \\
\hline $\begin{array}{l}\text { MEIS. Multi-factor } \\
\text { Emotional Intelligence } \\
\text { Scale (Mayer et al., 1999) }\end{array}$ & $\begin{array}{l}\text { Buena para la puntuación global } \\
\qquad(.70-.85) \text {. } \\
\text { Baja para ramas } 3 \text { y } 4 \text { (.35-.66) }\end{array}$ & $\begin{array}{c}\text { Pequeñas y moderadas } \\
\text { correlaciones con } \\
\text { inteligencia cristalizada. } \\
\text { Bajas correlaciones con los } \\
\text { Cinco Grandes }\end{array}$ & $\begin{array}{l}\text { Consenso, } \\
\text { experto y } \\
\text { objetivo }\end{array}$ & $\begin{array}{l}\text { No se conoce } \\
\text { ninguna. }\end{array}$ \\
\hline $\begin{array}{l}\text { MSCEIT. Mayer-Salovey- } \\
\text { Caruso Emotional } \\
\text { Intelligence Test (Mayer et } \\
\text { al., 2002) }\end{array}$ & $\begin{array}{c}\text { Buena para la puntuación total } \\
(.90) \\
\text { Rama 1: buena }(.89) \\
\text { Rama 2: buena }(.74) \\
\text { Rama 3: moderada }(.67) \\
\text { Rama 4: moderada-baja }(.60)\end{array}$ & $\begin{array}{c}\text { Convergencia entre criterios } \\
\text { de consenso y expertos. } \\
\text { Correlaciones muy bajas } \\
\text { con medidas de IE rasgo y } \\
\text { los Cinco Grandes. }\end{array}$ & $\begin{array}{l}\text { Consenso y } \\
\text { experto }\end{array}$ & $\begin{array}{l}\text { Extremera, } \\
\text { Fernández- } \\
\text { Berrocal y } \\
\text { Salovey } \\
(2006)\end{array}$ \\
\hline $\begin{array}{l}\text { TIEFBA. Test de } \\
\text { Inteligencia Emocional de } \\
\text { la Fundación Botín } \\
\text { (Fernández-Berrocal et al., } \\
\text { in press) }\end{array}$ & $\begin{array}{c}\text { Buenas puntuaciones remitidas } \\
\text { por los autores. } \\
\text { Rama 1: buena }(.86) \\
\text { Rama 1: buena }(.76) \\
\text { Rama 1: buena }(.76) \\
\text { Rama 1: buena }(.74) \\
\text { Puntuación global: buena }(.91) \\
\end{array}$ & $\begin{array}{l}\text { Correlaciones positivas con } \\
\text { empatía y negativas con } \\
\text { angustia. } \\
\text { Correlaciones no } \\
\text { significativas con rasgos de } \\
\text { personalidad. }\end{array}$ & Experto & $\begin{array}{l}\text { Versión } \\
\text { original } \\
\text { española. }\end{array}$ \\
\hline
\end{tabular}

Nota. Los datos se presentan de forma sucinta, para información más detallada consultar la fuente original.

Tabla 3. Instrumentos de evaluación de la percepción emocional y sus características

\begin{tabular}{l}
\hline Instrumento \\
\hline CARAT. Communication of Affect \\
Receiving Ability Test (Buck, 1976) \\
PONS. Profile of Nonverbal \\
Sensitivity (Rosenthal et al., 1979) \\
CFT. Chimeric Faces Test (Levine \\
\& Levy, 1986)
\end{tabular}

IPT. Interpersonal Perception Task (Costanzo \& Archer, 1989)

MTAP. Minnesota Test of Affective Processing (Lai et al., 1991)

DANVA2-AF. Diagnostic Assessment of Non Verbal AffectAdult Facial Expressions (Nowicki \& Carton, 1993)

CASP. The Child and Adolescent Social Perception Measure (MagillEvans et al., 1995)

DANVA2-AP. Diagnostic Assessment of Non Verbal AffectAdult Paralanguage (Baum \& Nowicki, 1998) FAB. Florida Affect Battery (Bowers et al., 1999)

JACBART. Japanese and Caucasian Brief Affect Recognition Test (Matsumoto et al., 2000) RAFL. Recognition of Affect in a Foreing Language (Scherer, sin publicar)

ERT. Emotion Recognition Test (Shimokawa et al., 2000) RMET. Reading the Mind in the Eyes Test (Baron-Cohen et al., 2001)

EARS. Emotional Accuracy

\begin{tabular}{|c|}
\hline Fiabilidad \\
\hline $\begin{array}{l}\text { Buena fiabilidad test- } \\
\text { retest }(.79 \text { a } .80)\end{array}$ \\
\hline Buena $(.86)$ \\
\hline Buena (.81) \\
\hline $\begin{array}{l}\text { Buena fiabilidad test- } \\
\text { retest }(.70)\end{array}$ \\
\hline $\begin{array}{l}\text { Baja consisten-cia } \\
\text { interna }(.52)\end{array}$ \\
\hline
\end{tabular}

Moderada-alta consistencia interna (.62-.77)

Buena fiabilidad testretest (.74-.84)

Buena consis-tencia interna, fiabilidad

test-retest y fiabilidad interratio (>.80)

Buena consistencia interna (.70-.78).

Buena fiabilidad testretest (.83)

Muy buena fiabilidad test-retest (.89-.97)

Muy buena (.86 a .92)

$$
?
$$

Fiabilidad test-retest variable (.43 a .82)

Fiabilidad test retest moderada (.63)

Buena (.80 para

$\begin{array}{ccc}\text { Validez } & \begin{array}{c}\text { Sistema de } \\ \text { puntuación }\end{array} & \text { Versión en español } \\ \begin{array}{c}\text { Se encuentran } \\ \text { correlaciones con } \\ \text { desarrollo profesional. }\end{array} & \text { Objetivo } & \begin{array}{c}\text { No se conoce } \\ \text { ninguna. }\end{array} \\ \begin{array}{c}\text { Correlaciones con áreas de } \\ \text { inteligencia y rendimiento. }\end{array} & \text { Experto } & \begin{array}{c}\text { Martínez-Sánchez } \\ \text { et al. (2013) } \\ \text { No se conoce } \\ \text { ninguna. }\end{array} \\ & \text { Objetivo } & \text { n }\end{array}$

\section{Correlaciona con}

habilidades sociales y con pruebas similares.

Correlaciona con otras medidas de habilidad similares.

Correlaciona con éxito y conducta social.

Hay correlaciones en diferentes grupos de edad y grupos diagnósticos.

Correlaciona con rendimiento académico, visión espacial y pruebas similares, no con CI.

?
Correlaciones bajas con
los Cinco Grandes.

$$
?
$$

Correlaciones con capacidad intelectual.

Correlaciona con CI.

Pequeñas correlaciones
Objetivo

García, Fuentes et al. (2003)

Objetivo

No se conoce ninguna.

No se conoce ninguna.

No se conoce
ninguna.

Objetivo No se conoce ninguna.

\section{Objetivo No se conoce ninguna.}

Objetivo No se conoce ninguna.

Objetivo No se conoce ninguna.

Objetivo No se conoce ninguna.

Objetivo Perez-Sayes, Luna y Tirapu, (2009)

Consenso y No se conoce 
Research Scales (Geher et al., 2001)

Emotional Stroop (Sharma \& McKenna, 2001)

FEFA. Frankfurt Test and Training of Social Affect (Bölte et al., 2002)

EMT. Emotion Matching Task (Izard et al., 2003)

ERT. Emotional Recognition TestRevised (Lee et al., 2004)

DANVA2-POS. Diagnostic Analysis of Nonverbal Acuracy 2 Posture Test (Pitterman \& Nowiki, 2004)

EIT. Emotional Inspection Time (Austin, 2005)

CATS. Comprehensive Affect Testing System (Froming et al., 2006)

The NimStim set of facial expresions (Tottenham et al., 2009) MERT. Multimodal Emotion Recognition Test (Bänziger et al., 2009)

ERI. Emotion Recognition Index (Scherer \& Scherer, 2011)

STJ-based measure of EI. Situational Judgment Test-based measure of Emotional Intelligence (Sharma et al., 2013) puntuación expertos y .75 para consenso) $?$

Muy buena consistencia interna (.91-.95) y fiabilidad test-retest (.89-.92)

Variable por subescalas (.54-.80); buena puntuación total (.81) $?$

Buena consistencia interna (.68-.77) y fiabilidad test-retest (.85)

con autoinformes de empatía.

$$
?
$$

Correlaciona con instrumentos similares.

Correlaciona con otras medidas de conocimiento emocional

\section{$?$}

Baja correlación con otras capacidades no verbales.

Correlaciones con

competencia social y personalidad.

Correlaciones bajas con inteligencia y pruebas similares.

\section{Buena (.84)}

Buena (.78)

?

Buena consistencia interna (.71 a .82)

Fiabilidad test-retest (.82)

Correlaciona con pruebas similares.

Validez convergente alta (.67-.84) con instrumentos similares.

Correlaciona con pruebas similares.

Muy bajas con los Cinco Grandes

Correlaciona con satisfacción vida y rendimiento. Bajas

correlaciones con personalidad y muy bajas objetivo

Objetivo

Objetivo

Objetivo

Alonso-Alberca et al. (2012)

Objetivo

No se conoce ninguna.

Objetivo

No se conoce ninguna.

Objetivo

No se conoce ninguna.

Disponible en http://www.psychol ogysoftware.com/C ATS.htm con IE rasgo
No se conoce ninguna.

No se conoce ninguna.

Objetivo y No se conoce

Experto ninguna.

Experto

No se conoce ninguna.

Nota. Los datos se presentan de forma sucinta, para información más detallada consultar la fuente original. (?): no se han obtenido datos al respecto o no son suficientes para su interpretación.

de percepción emocional. Entre los instrumentos parciales de evaluación de la IE capacidad destinados a la medida de las capacidades propias de la faceta de comprensión emocional encontramos los presentados junto a sus características propias en la Tabla 4. Por último, se presentan en la Tabla 5 los instrumentos parciales de IE capacidad cuyo objetivo es la medida de la faceta correspondiente a la última rama del modelo de IE capacidad: la regulación emocional.

\section{Discusión}

Aparentemente, se dispone de gran cantidad y variedad de pruebas de IE capacidad. No obstante, de nuestro análisis se desprende como más bien deficiente el conjunto de instrumentos actualmente disponibles para la medida de la IE capacidad, lo que es congruente con lo reconocido recientemente por Mayer et al. (2016) al respecto.

En primer lugar, la presencia de medidas comprehensivas en la literatura es bastante limitada. Por su parte, encontramos un gran número de medidas parciales. Sin embargo, una visión analítica de los instrumentos pertenecientes a esa inmensa mayoría de medidas parciales, nos permite ver de forma instantánea que la representatividad de las ramas de la IE capacidad entre todos estos instrumentos no es, en absoluto, homogénea. Tal y como se observa, se ha producido un amplio desarrollo de instrumentos destinados a la medición de las capacidades referentes a la rama de percepción, expresión e identificación de emociones, que suponen más del $75 \%$ de los instrumentos recogidos, y cuyo 
Tabla 4. Instrumentos de evaluación de la comprensión emocional y sus características

\begin{tabular}{|c|c|c|c|c|}
\hline Instrumento & Fiabilidad & Validez & $\begin{array}{l}\text { Sistema de } \\
\text { puntuación }\end{array}$ & $\begin{array}{c}\text { Versión en } \\
\text { español }\end{array}$ \\
\hline $\begin{array}{l}\text { LEAS. Levels of Emotional } \\
\text { Awareness Scale (Lane et al., } \\
\text { 1990) }\end{array}$ & Buena (0.81) & $\begin{array}{l}\text { No presenta correlación con los } \\
\text { Cinco Grandes. }\end{array}$ & Objetivo & $\begin{array}{c}\text { Disponible en } \\
\text { https://eleastest.ne } \\
\mathrm{t}\end{array}$ \\
\hline $\begin{array}{l}\text { Emotion differentiation card- } \\
\text { sorting task (Kang et al., 2005) }\end{array}$ & $?$ & 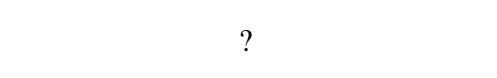 & Objetivo & $\begin{array}{l}\text { No se conoce } \\
\text { ninguna. }\end{array}$ \\
\hline $\begin{array}{l}\text { STEU. Situational Test of } \\
\text { Understanding (MacCann y } \\
\text { Roberts, 2008) }\end{array}$ & Moderada (.72) & $\begin{array}{c}\text { Validez predictiva en capacidad } \\
\text { verbal (vocabulario). } \\
\text { Validez incremental en satisfacción } \\
\text { con la vida y rendimiento académico. } \\
\text { Bajas correlaciones con los Cinco } \\
\text { Grandes. }\end{array}$ & Experto & $\begin{array}{l}\text { Cejudo y Pérez- } \\
\text { González (2013) }\end{array}$ \\
\hline $\begin{array}{l}\text { STJ-based measure of EI. } \\
\text { Situational Judgment Test- } \\
\text { based measure of Emotional } \\
\text { Intelligence (Sharma et al., } \\
\text { 2013) }\end{array}$ & $\begin{array}{l}\text { Buena } \\
\text { consistencia } \\
\text { interna }(.71 \text { a } .82) \\
\text { Fiabilidad test- } \\
\text { retest }(.82)\end{array}$ & $\begin{array}{c}\text { Validez predictiva para satisfacción } \\
\text { con la vida y rendimiento académico. } \\
\text { Bajas correlaciones con los Cinco } \\
\text { Grandes. } \\
\text { Correlaciones muy bajas con medidas } \\
\text { de IE rasgo (TEIQue). }\end{array}$ & Experto & $\begin{array}{l}\text { No se conoce } \\
\text { ninguna. }\end{array}$ \\
\hline
\end{tabular}

Tabla 5. Instrumentos de evaluación de la regulación emocional y sus características

\begin{tabular}{|c|c|c|c|c|}
\hline Instrumento & Fiabilidad & Validez & $\begin{array}{l}\text { Sistema de } \\
\text { puntuación }\end{array}$ & $\begin{array}{c}\text { Versión en } \\
\text { español }\end{array}$ \\
\hline $\begin{array}{l}\text { MP-EMA. Maximum-Performance } \\
\text { Emotional Management Abilities } \\
\text { (Freudenthaler \& Neubauer, 2005) }\end{array}$ & $\begin{array}{l}\text { Consistencia } \\
\text { interna } \\
\text { moderada- } \\
\text { baja }(.43 \text { a } \\
.67)\end{array}$ & $\begin{array}{l}\text { Correlaciona con capacidad verbal. } \\
\text { No se encuentran relaciones con Cinco } \\
\text { Grandes. }\end{array}$ & Experto & $\begin{array}{l}\text { No se } \\
\text { conoce } \\
\text { ninguna. }\end{array}$ \\
\hline $\begin{array}{l}\text { STEM. Situational Test of } \\
\text { Emotion Management (MacCann } \\
\& \text { Roberts, 2008) }\end{array}$ & $\begin{array}{l}\text { Moderada } \\
\quad(.68)\end{array}$ & $\begin{array}{c}\text { Validez predictiva en capacidad verbal } \\
\text { (vocabulario) } \\
\text { Validez incremental en satisfacción con la } \\
\text { vida y rendimiento académico. } \\
\text { Bajas correlaciones con los Cinco } \\
\text { Grandes. }\end{array}$ & Experto & $\begin{array}{l}\text { Rodrigo- } \\
\text { Ruiz (2017) }\end{array}$ \\
\hline
\end{tabular}

resultado nos informa única y exclusivamente del nivel del sujeto en la primera rama del modelo de Mayer y Salovey (1997; Mayer et al., 2016). A pesar de ello, la evaluación de esta rama 1 del modelo de IE capacidad es incompleta, en tanto en cuanto apenas disponemos de instrumentos centrados en evaluar aspectos de la percepción emocional, pero por el contrario la faceta de expresión emocional es obviada. Puesto que esta primera rama del modelo de IE capacidad es la que contiene las capacidades básicas y elementales sobre las que se desarrollan aquellas más complejas (e.g., Joseph \& Newman, 2010), entendemos lo que una medida de estas capacidades nos ofrece dista mucho de ser una evaluación del constructo IE capacidad.

Si centramos nuestra atención en la cuarta rama del modelo expuesto por Mayer y Salovey (1997), la rama de regulación emocional, aquella que refleja la adquisición de las capacidades más complejas y de un nivel más elevado dentro del modelo, podemos detectar la poca representatividad que los instrumentos que realizan medición sobre esta faceta tienen en el desarrollo de medidas de IE capacidad. Parece lógico esperar que aquello que más información ofrece sobre la IE capacidad sea lo más explotado e investigado, sin embargo, los datos muestran una tendencia completamente contraria con una presencia ingentemente mayor de pruebas referentes a las capacidades más básicas.

A continuación, analizaremos los resultados obtenidos tanto para instrumentos comprehensivos como parciales y en cada una de las facetas de la IE capacidad. 


\section{Instrumentos comprehensivos de evaluación de IE capacidad}

Los instrumentos comprehensivos engloban aquellos que realizan una medición comprehensiva de la IE capacidad a través de todas las facetas que la componen. Como cabe esperarse, se trata de una evaluación que permite la obtención de una información más representativa de la IE capacidad del individuo, por cuanto cuenta con datos del perfil multifacético completo.

Son tres los instrumentos comprehensivos de IE capacidad que encontramos en la literatura. En primer lugar encontramos el Multi-factor Emotional Intelligence Scale (MEIS; Mayer, Caruso, \& Salovey, 1999). Hallamos también una versión actualizada y mejorada del mismo, al que se ha denominado Mayer-Salovey-Caruso Emotional Intelligence Test (MSCEIT; Mayer et al., 2002), y en el que se solucionan algunos de los principales problemas presentados por el MEIS. Por último, encontramos un último instrumento del que por el momento tenemos poca información, el Test de Inteligencia Emocional de la Fundación Botín (TIEFBA; FernándezBerrocal et al., 2018), desarrollado en lengua española para población adolescente y que sigue, como los anteriores, el modelo de cuatro ramas propuesto por Mayer y Salovey (1997).

Observamos que dos de las pruebas a las que se ha hecho constancia (i.e., MEIS y MSCEIT), son en realidad dos versiones de una misma, por lo que el uso de la primera ha quedado relegado a un segundo plano tras la aparición de la segunda. A pesar de ello, se ha considerado aquí como una herramienta más de la evaluación de la IE capacidad de forma comprehensiva, sabiendo en realidad que no constituye una alternativa práctica para la medición de la misma.

Como puede comprobarse en la Tabla 2, las propiedades psicométricas del MEIS respaldan moderadamente su utilidad. Si bien es cierto que la fiabilidad para la prueba percibida de forma global es buena, los índices estadísticos no ofrecen noticias satisfactorias en lo referente a las ramas superiores del modelo, las cuales obtienen no muy elevados índices de fiabilidad, lo que no supone un soporte estable para el modelo de cuatro ramas.
Si hablamos de índices estadísticos, tanto la fiabilidad como la validez del MSCEIT permiten que califiquemos al instrumento como aceptable, aunque el problema que observábamos en el MEIS continúa afectando al MSCEIT; pese a que la fiabilidad total es buena, las ramas superiores del modelo no disfrutan de una fiabilidad tan buena como las ramas inferiores, lo cual ha de afectar necesariamente a la fidelidad de la información sobre la IE del sujeto de la que nos aporta este el instrumento. Está disponible una versión en español del MSCEIT realizada por Extremera, Fernández-Berrocal y Salovey (2009) validada por una muestra de estudiantes universitarios que reporta buenos resultados en fiabilidad y consistencia interna (.63 a .90), altas correlaciones entre los métodos de corrección y correlaciones con edad (Extremera et al., 2009), puntuaciones muy similares a la versión original.

Son, sin embargo, tanto el MEIS como el MSCEIT, instrumentos que han recibido importantes críticas por parte de la comunidad científica. Entre las principales, encontramos las referidas a los índices estadísticos; algunos autores (e.g., Brannick, Wahi, \& Goldin, 2011; Maul, 2012; Kong, 2014) informan de una validez menor del MSCEIT frente a la que refieren los autores. Ciertos autores (Fiori \& Antonakis, 2012; Maul, 2012; Rode et al., 2008) están de acuerdo en que definitivamente aquello que mide el MSCEIT no se refiere al constructo de IE tal y como lo entendemos. Su sistema de puntuación es también problemático y atípico en la evaluación de una inteligencia (e.g., Brody, 2004; Maul, 2012; Fiori et al., 2014; Føllesdal \& Hagtvet, 2009). Asimismo, los análisis factoriales realizados al respecto (Brannick et al., 2011; Fan, Jackson, Yang, Tang, \& Zhang, 2010; Legree et al., 2014; Roberts et al., 2006; Rode et al., 2008) aportan diferentes evidencias empíricas sobre la incongruencia entre la estructura del instrumento y la del modelo teórico sobre la que se sustenta, pues es habitual la constatación de tres factores en lugar de cuatro, quedando la rama 2 del modelo (utilización o facilitación) sin factor estadístico propio

En cuanto al TIEFBA, es poco lo que sabemos debido a su novedad, puesto que, hasta el momento, únicamente tenemos acceso a la información facilitada por los autores en su primer 
trabajo publicado con este instrumento. Futuros trabajos irán proporcionando información sobre su funcionamiento y características psicométricas. Por el momento, entendemos que el sistema de puntuación realizado por consenso de un grupo de 22 expertos podría suponer cierta problemática, aunque su aparición puede suponer una buena alternativa al uso del MSCEIT.

\section{Instrumentos parciales de evaluación de IE capacidad}

Es importante puntualizar que una evaluación parcial no es equivalente a una evaluación comprehensiva puesto que no obtendremos una muestra representativa del conjunto de facetas que integran la IE capacidad. No obstante, puede resultar una evaluación pertinente y de gran utilidad para obtener información acerca de la faceta que deseamos evaluar y que presumiblemente correlacionaría positivamente con la IE capacidad global. Pese a que algunos estudios han evidenciado que existe cierta relación entre, por ejemplo, la identificación de emociones y una mejor regulación emocional (e.g., Gohm \& Clore, 2000; 2002; Mennin, Holaway, Fresco, Moore, \& Heimberg, 2007), otros nos recuerdan que no siempre puede predecirse la IE a partir de ciertas facetas de las misma (O'Sullivan, 2007), por lo que el tratamiento e interpretación de los datos obtenidos a través de instrumentos parciales debe realizarse con mucha precaución. Evaluar una parte de la IE no es, en cualquier caso, evaluar la IE.

\section{Instrumentos de evaluación de la percepción emocional (P)}

La percepción emocional es sin duda alguna la faceta más estudiada de la IE y aquella de la que más instrumentos de medida disponemos (e.g., Rodrigo-Ruiz, Pérez-González, \& Cejudo, 2017). No obstante, no está claro aún que la medición se esté realizando de forma correcta, ya que no existen criterios válidos para atribuir la categoría de "correcto" a una de las opciones de respuesta dada (Rivers, Brackett, Salovey, \& Mayer, 2007).

Existen diferencias importantes en las tareas a realizar en las diferentes pruebas de percepción emocional. Si bien es cierto que la mayoría de ellas se centran en tareas de reconocimiento de emociones en expresiones faciales (e.g., JACBART; Matsumoto et al., 2000), otras utilizan como estímulo grabaciones de voz (e.g., DANVA2-AP; Baum \& Nowicki, 1998) o posturas corporales (e.g., PONS; Rosenthal, Hall, DiMatteo, Rogers y Archer, 1979), e incluso comportamientos no verbales (e.g., IPT; Costanzo \& Archer, 1989); alguna utiliza asimismo tareas de reconocimiento de emociones en descripciones escritas (i.e., EARS; Geher, Wagner, \& Brown, 2001).

También cabe destacar que la mayoría de las pruebas de evaluación de percepción emocional son pruebas de "identificación" y no de "discriminación". Tal y como han explicado Rodrigo-Ruiz et al. (2017), las pruebas de identificación están sujetas al sesgo de respuesta de tendencia a responder repetidamente con la misma emoción ante cualquier estímulo presentado.

$\mathrm{Si}$ atendemos a los niveles de fiabilidad y validez de cada una de las pruebas (ver Tabla 4) podemos constatar que, efectivamente, los datos se corresponden con los de unos instrumentos generalmente fiables y con cierta validez, y psicométricamente adecuados. Los instrumentos recogidos en el presente trabajo tienen en términos generales una fiabilidad que en muchos casos oscila entre .80 y .90 , lo cual es una fiabilidad adecuada tanto para la investigación como para la medición en sujetos. No obstante, si afirmamos que estas pruebas realizan una medición de la misma área, deberían correlacionar entre sí, lo que no siempre ocurre u ocurre con correlaciones muy pequeñas (Mayer et al., 2008). Tal y como informa Hall (2001), aunque existen excepciones, la mayoría de este tipo de pruebas presenta una correlación de entre -.10 y .20, lo que no puede ser considerado como un dato a favor de las mismas. De forma similar, Roberts et al. (2006) informan de correlaciones inexistentes entre ciertas medidas de percepción emocional y la escala de percepción del MSCEIT, a pesar de que se encuentran correlaciones bajas con otras escalas de este mismo instrumento. Mayer et al. (2008) realizan una distinción entre medidas basadas en capacidades específicas y medidas basadas en modelos integradores tratando de explicar esta posible independencia, no obstante, 
la escasa correlación existente sigue sin encontrar una explicación aceptable a día de hoy.

Otro factor a tener en cuenta es si realmente estas pruebas están consiguiendo informarnos acerca de niveles de percepción emocional entendida como parte de la IE. Puede resultar evidente la importancia de conocer e identificar las expresiones faciales $\mathrm{u}$ otros estímulos no verbales como una capacidad más de esta faceta de la IE, no obstante, como señala O'Sullivan (2007), esto no nos garantiza en absoluto que el sujeto evaluado entienda en efecto las emociones que está experimentando el otro.

En cuanto a las traducciones al español, si bien es cierto que encontramos pocos instrumentos adaptados para su uso en nuestro idioma, sí encontramos algunos, que coinciden con los instrumentos mencionados como más utilizados en la literatura científica.

\section{Instrumentos de evaluación de la facilitación emocional (F)}

Se trata ésta de la rama del modelo con menos presencia en investigación, no se encuentra en ella ningún instrumento de medida que aluda a la evaluación de la capacidad de facilitación emocional. Recuérdese que Izard et al. (2007) consideran la facilitación o utilización emocional como parte de la regulación emocional, por lo que según estos autores no tiene sentido concebirla como una entidad propia independiente de la rama 4 de la IE capacidad.

A pesar de que algunos autores (Zeidner, Matthews, \& Roberts, 2009) han considerado al Emotional Stroop Task (Mogg, Matthews, Bird, \& MacGregor-Morris, 1990) como una medida que podría utilizarse para la evaluación de la facilitación emocional, hemos descartado su inclusión por tratarse en realidad de una prueba de inhibición de respuesta automática, que poco tiene que ver con la evaluación de la facilitación emocional. Más aún, autores que han realizado otras de las principales clasificaciones $\mathrm{o}$ recopilaciones de instrumentos sobre IE (MacCann, Matthews, Zeidner, \& Roberts, 2004; Pérez-González et al., 2007; Rivers et al., 2007; Siegling et al., 2015), tampoco lo han considerado.
Instrumentos de evaluación de la comprensión emocional (U)

Como el lector puede observar, apenas se dispone de algunos instrumentos de esta tercera faceta de la IE. Tanto la fiabilidad como la validez observadas nos permiten hablar con cierta holgura de pruebas aceptables para la investigación y la medida de la comprensión emocional en sujetos.

Vemos en este caso que se dispone de una importante representación de estas medidas en lengua española, ya que de los cuatro instrumentos de la Tabla 4, al menos dos de ellos se han traducido y adaptado a nuestra lengua. Para investigadores interesados específicamente en esta rama en el ámbito infantil nos sumamos a la recomendación de Pérez-González y Qualter (2018) de considerar el protocolo de entrevista KAI-R (Kusche, Greenberg, \& Beilke, 1998), el cual constituye un interesante instrumento para evaluación la comprensión emocional en la infancia.

\section{Instrumentos de evaluación de la regulación emocional (R)}

En esta última categoría nos encontramos con tan sólo dos instrumentos, entre los que, uno de ellos tiene una versión adaptada a la lengua y sociedad española.

Queda reflejado en la Tabla 5 que los datos estadísticos que apoyan la validez $y$, especialmente, la fiabilidad de estas pruebas, no resultan una garantía segura. No se han conseguido aún resultados óptimos en la fiabilidad de los instrumentos. Parece ser que, hasta el momento, el instrumento que mejores resultados ha conseguido al respecto es el STEM (MacCann \& Roberts, 2008), con unos índices de fiabilidad en torno a .68. La versión española de dicho test supera dicho índice de fiabilidad con un $\alpha=.80$ (Rodrigo-Ruiz, 2017), lo que constituye una buena fiabilidad.

Una de las principales dificultades adheridas a este tipo de pruebas es la dificultad de puntuar los resultados. El mismo problema del que ya hemos hablado para las medidas de IE capacidad en general se acentúa más si cabe en un tipo de tarea que resulta muy difícil de identificar una respuesta correcta. 


\section{Conclusiones}

A pesar de que los resultados iniciales anunciaban la existencia de un gran número de instrumentos de IE capacidad, un análisis pormenorizado de los mismos nos muestra una gran carencia en el desarrollo de dichas medidas. Encontramos, por un lado, dos únicos instrumentos vigentes para la realización de una medición comprehensiva (MSCEIT; Mayer et al., 2002; TIEFBA, Fernández-Berrocal et al., 2018), el primero con muchas críticas sobre su construcción y funcionamiento, y el segundo con apenas información debido a su novedad. Asimismo, hallamos una importante variedad de instrumentos de medición parcial entre los que destacan en número aquellos que miden facetas más básicas de la IE capacidad, disponiendo de muy pocos instrumentos adecuados para la medida de las capacidades superiores de la IE capacidad.

Como se ha venido viendo en el análisis de los diferentes instrumentos de IE capacidad, nos encontramos ante un conjunto de instrumentos formados por dos grupos bien diferenciados principalmente. En primer lugar, encontramos aquellos provenientes de otras áreas afines, aunque no construidos específicamente para el estudio de la IE; en segundo lugar, obtenemos en nuestra búsqueda instrumentos propios de la medida de la IE que se encuentran aún en su fase experimental o en desarrollo.

Los resultados psicométricos, aunque nos permiten acercarnos poco a poco a un constructo bastante desconocido aún, indican la poca adecuación de las medidas disponibles para una evaluación óptima de la IE capacidad. En primer lugar, la fiabilidad de los instrumentos no es precisamente un dato esperanzador, puesto que en muchos casos, especialmente en medidas que evalúan las ramas más altas del modelo de IE capacidad, la fiabilidad roza los límites de la aceptación. Si hablamos de validez, el problema permanece, puesto que la validez de los instrumentos de IE capacidad es generalmente moderada (Matthews, Zeidner, \& Roberts, 2012). En cuanto a la convergencia entre los diferentes instrumentos, los datos no son favorables, puesto que, en general, y especialmente entre instrumentos de la rama 1 del modelo, de los que poseemos más resultados, las correlaciones son muy bajas o inexistentes (Mayer et al., 2008). Todo ello invita al uso prudente de estos instrumentos y la interpretación de sus resultados con la mayor precaución posible.

Entre las limitaciones del presente trabajo cabe destacar la naturaleza no sistemática de la revisión, lo cual podría acarrear la pérdida de posible información que habría sido fácilmente recogida mediante otros métodos de búsqueda. Debido a la magnitud de la búsqueda realizada, entendemos que la ausencia de posibles pruebas a incluir es mínima, no obstante posible. En el momento actual, en todo caso, y desde hace ya algunos años, el instrumento más extendido y con un uso más generalizado en la evaluación de la IE capacidad es el MSCEIT (Fiori et al., 2014; Mayer et al., 2008), pese a que su correcto funcionamiento, como ya se ha mencionado anteriormente, está en entredicho.

Como puede comprobarse, la situación actual abre las puertas a la investigación y desarrollo de nuevas medidas que aseguren una medición acorde a la realidad. Los instrumentos en desarrollo y las nuevas aproximaciones necesarias han de otorgar a las medidas de IE capacidad la validez necesaria para conocer el nivel de adquisición de las capacidades involucradas para la superación de las diferentes tareas propuestas y comprobar el impacto que los diferentes métodos de puntuación empleados ejercen sobre los resultados finales de las pruebas que utilizamos en la actualidad.

\section{Referencias}

Alonso-Alberca, N., Vergara, A.I., FernándezBerrocal, P., Johnson, S. R., \& Izard, C. E. (2012). The adaptation and validation of the Emotion Matching Task for preschool children in Spain. International Journal of Behavioral Development, 36(6), 489-494. doi:10.1177/0165025412462154

Austin, E. J. (2005). Emotional intelligence and emotional information processing. Personality and Individual Differences, 39, 403-414. doi:10.1016/j.paid.2005.01.017

Bänziger, T., Grandjean, D., \& Scherer, K.R. (2009). Emotion recognition from expressions 
in face, voice, and body: The Multimodal Emotion Recognition Test (MERT). Emotion, 9(5), 691-704. doi:10.1037/a0017088

Baron-Cohen, S., Wheelwright, S., Hill, J., Raste, Y., \& Plumb, I. (2001). The "reading the mind in the eyes" test revised version: A study with normal adults, and adults with Asperger Syndrome or high-functioning autism. Journal of Child Psychology and Psychiatry, 42, 241-251. doi:10.1017/s0021963001006643

Baum, K. M., \& Nowicki, S. (1998). Perception of emotion: Measuring decoding accuracy of adult prosodic cues varying intensity. Journal of Nonverbal Behavior, 22(2), 89-107. doi:10.1023/a:1022954014365

Bisquerra, R., Pérez González, J. C., \& García Navarro, E. (2015), Inteligencia emocional en educación. Madrid: Ed. Síntesis.

Bölte, S., Feineis-Matthews, S., Leber, S., Dierks, T., Hubl, D., \& Poustka, F. (2002). The development and evaluation of a computerbased program to test and to teach the recognition of facial affect. International Journal of Circumpolar Health, 61(2), 61-68. doi:10.3402/ijch.v61i0.17503

Bowers, D., Blonder, L. X., \& Heilman, K. M. (1999). Florida Affect Battery. Gainesville, FL: University of Florida.

Brannick, M. T., Wahi, M. M., \& Goldin, S. B. (2011). Psychometrics of Mayer-SaloveyCaruso Emotional Intelligence Test (MSCEIT) scores. Psychological Reports, 109(1), 327-337. doi:10.2466/03.04.pr0.109.4.327-337

Brody, N. (2004). What cognitive intelligence is and what emotional intelligence is not. Psychological Inquiry, 15, 234-238.

Buck, R. (1976). A test of nonverbal receiving ability: Preliminary studies. Human Comunication Research, 2, 162-171. doi:10.1111/j.1468-2958.1976.tb00708.x

Buck, R. Powers, S. R., \& Hull, K. S. (2017). Measuring emotional and cognitive empathy using dynamic, naturalistic, and spontaneous emotion displays. Emotion, 17(7), 1120-1136. doi:10.1037/emo0000285

Cejudo, J., \& Pérez-González, J. C. (2013). Adaptación y traducción del Situational Test of Understanding - Adolescents Short Form (STEU-ASF). Trabajo inédito.
Costanzo, M., \& Archer, D. (1989). Interpreting the expressive behaviour of others: The Interpersonal Perception Task. Journal of Nonverbal Behaviour, 13, 225-245. doi:10.1007/bf00990295

Cronbach, L. J. (1949). Essentials of psychological testing. New York: Harper \& Row.

Extremera, N., Fernández-Berrocal, P., \& Salovey, P. (2006). Spanish version of the Mayer-Salovey-Caruso Emotional Intelligence Test (MSCEIT). Version 2.0: reliabilities, age and gender differences. Psicothema, 18, 4248.

Fan, H., Jackson, T., Yang, X., Tang, W., \& Zhang, J. (2010). The factor structure of the Mayer-Salovey-Caruso Emotional Intelligence Test V 2.0 (MSCEIT): A meta-analytic structural equation modeling approach. Personality and Individual Differences, 48, 781-785. doi:10.1016/j.paid.2010.02.004

Fernández-Berrocal, P., \& Extremera, N. (2016). Ability emotional intelligence, depression, and well-being. Emotion Review, 8(4), 311315. doi:10.1177/1754073916650494

Fernández-Berrocal, P., Ruiz-Aranda, D., Salguero, J.M., Palomera, R., \& Extremera, N. (2018). La relación del Test de Inteligencia Emocional de la Fundación Botín (TIEFBA) con el ajuste personal y escolar de adolescentes españoles. Revista de Psicodidáctica, $\quad$ 23(1), $1-8$. doi:10.1016/j.psicod.2017.07.001

Fiori, M., \& Antonakis, J. (2012). Selective attention to emotional stimuli: What IQ and openness do, and emotional intelligence does not. Intelligence, 40, 245-254.

doi:10.1016/j.intell.2012.02.004

Fiori, M., Antonietti, J. P., Mikolajczak, M., Luminet, O., Hansenne, M., \& Rossier, J. (2014). What is the ability emotional intelligence test (MSCEIT) good for? An evaluation using item response theory. PLOS ONE, 9(6), e98827. doi:10.1371/journal.pone.0098827

Føllesdal, H., \& Hagtvet, K. A. (2009). Emotional intelligence: The MSCEIT from the perspective of generalizability theory. Intelligence, 37(1), 94-105. doi:10.1016/j.intell.2008.08.005 
Freudenthaler, H. H., \& Neubauer, A.C. (2005). Emotional intelligence: The convergent and discriminant validities of intra- and interpersonal emotional abilities. Personality and Individual Differences, 39, 569-579. doi:10.1016/j.paid.2005.02.004

Froming, K. B., Levy, C. M., Schaffer, S. G., \& Ekman, P. (2006). The Comprehensive Affect Testing System. Psychology Software, Inc.

García, S., Fuentes, I., Ruiz, J.C., Gallach, E., y Roder, V. (2003). Application of the IPT in a Spanish sample: Evaluation for the social perception subprogramme. International Journal of Psychology and Psychological Therapy, 3(2), 299-310.

Geher, G., Warner, R. M., \& Brown, A. S. (2001). Predictive validity of the emotional acuracy research scale. Intelligence, 29, 373-388. doi:10.1016/s0160-2896(00)00045-3

Gohm, C. L., \& Clore, G. L. (2000). Individual differences in emotional experience: Mapping available scales to processes. Personality and Social Psychology Bulletin, 26, 679-697. doi:10.1177/0146167200268004

Gohm, C. L., \& Clore, G. L. (2002). Four latent traits of emotional experience and their involvement in well-being, coping, and atributional style. Cognition and Emotion, 16, 495-518. doi:10.1080/02699930143000374

Guaita, V. L. (2007). Aspectos emocionales de la comunicación en niños en riesgo por pobreza extrema. Revista Iberoamericana de Diagnóstico y Evaluación Psicológica - e Avaliação Psicológica, 24(2), 177-192.

Hall, J. A. (2001). The PONS Test and the psychometric approach to measuring interpersonal sensitivity. En J. A. Hall y F. J. Bernieri (Eds.), Interpersonal Sensitivity: Theory and Measurement (pp. 143-60). Mahwah, NJ: Erlbaum.

Hodzic, S., Scharfen, J., Ripoll, P., Holling, H., \& Zenasni, F. (2017). How efficient are emotional intelligence trainings:a metaanalysis. Emotion Review, 73, 44-49. doi: $10.1177 / 1754073917708613$

Izard, C. E., Haskins, F. W., Schultz, D., Trentacosta, C. J., \& King, K. A. (2003). Emotion matching test. Trabajo inédito. Human Emotions Lab, Department of
Psychology, University of Delaware, Delaware, USA.

Izard, C. E., Trentacosta, C. J., King, K. A., Morgan, J. K., \& Diaz, M. (2007). Emotions, emotionality, and intelligence in the development of adaptive behavior. En G. Matthews, M. Zeidner, y R. Roberts (Eds.), Science of emotional intelligence: knowns and unknowns (pp. 101-150). Cambridge, MA: Oxford University Press.

Joseph, D. L., \& Newman, D. A. (2010). Emotional intelligence: An integrative metaanalysis and cascading model. Journal of Applied Psychology, 95(1), 54-78. doi:10.1037/a0017286

Kang, S., Day, J. D., \& Meara, N. M. (2005). Social intelligence and emotional intelligence: Starting a conversation about their similarities and differences. En R. Schulze, y R.D. Roberts (Eds.), Emotional intelligence: An international handbook (pp. 91-105). Cambridge, MA: Hogrefe \& Huber.

Kong, D. T. (2014). Mayer-Salovey-Caruso Emotional Intelligence Test (MSCEIT/ MEIS) and overall, verbal, and nonverbal intelligence: Meta-analytic evidence and critical contingences. Personality and Individual Differences, 66, 171-175. doi:10.1016/j.paid.2014.03.028

Kusche, C. A, Greenberg, M. T., \& Beilke, B. (1988). The Kusche affective interview. Trabajo inédito. Department of Psychology, University of Washington, Seattle, WA.

Lai, Z., Hughes, S., \& Shapiro, E. (1991). Manual for the Minnesota Tests of Affective Processing (MNTAP). Minnesota: University of Minnesota.

Lane, R. D., Quinlan, D. M., Schwartz, G. E., Walker, P. A., \& Zeitlin, S. B. (1990). The levels of emotional awareness scale: A cognitive-developmental measure of emotion. Journal of Personality Assessment, 55, 12434. doi:10.1207/s15327752jpa5501\&2_12

Lee, S. J., Miller, H. A., \& Moon, J. (2004). Exploring the forensic use of the emotional recognition test (ERT). International Journal of Offender Therapy and Comparative Criminology, 48(6), 664-82. doi:10.1177/0306624x04266278 
Legree, P. J., Psotka, J., Robbins, J., Roberts, R. D., Putka, D. J., \& Mullins, H. M. (2014). Profile similarity metrics as an alternate frameworks to score rating-based test: MSCEIT reanalyses. Intelligence, 47, 159174. doi:10.1016/j.intell.2014.09.005

Levine, S. C., \& Levy, J. (1986). Perceptual asymmetry for chimeric faces across the life span. Brain and Cognition, 5(3), 291-306. doi:10.1016/0278-2626(86)90033-3

MacCann, C., Joseph, D. L., Newman, D. A., \& Roberts, R. D. (2014). Emotional intelligence is a second-stratum factor of intelligence: Evidence from hierarchical and bifactor models. Emotion, 14, 358-374. doi: $10.1037 / \mathrm{a} 0034755$

MacCann, C., Matthews, G., Zeidner, M., \& Roberts, R. (2004). The assessment of emotional intelligence: On frameworks, fissures and the future. En G. Geher, (Ed.), Measuring Emotional Intelligence. Common Ground and Controversy (pp. 20-52). New York: Nova Science Publishes Inc.

MacCann, C., \& Roberts, R. D. (2008). New paradigms for assesing emotional intelligence: Theory and data. Emotion, 8(4), 540-551. doi: $10.1037 / \mathrm{a} 0012746$

Magill-Evans, J., Koning, C., Cameron-Sadava, A., \& Manyk, K. (1995). The Child and Adolescent Social Perception Measure. Journal of Nonverbal Behavior, 19, 151-169. doi:10.1007/bf02175502

Martínez-Sánchez, F., Fernández-Abascal, E. G., \& Martínez-Modia, J. C. (2013). Adaptación española de la versión reducida multicanal del Perfil de Sensibilidad No Verbal (MiniPONS). Anales de Psicología, 29(2), 604-613. doi:10.6018/analesps.29.2.161851

Martorell, C. (2005). Conferencias del V Congreso Iberoamericano de Evaluación Psicológica. Inteligencia emocional: realidad o falacia. Revista Iberoamericana de Diagnóstico y Evaluación Psicológica - e Avaliação Psicológica, 20(2), 165-182.

Matsumoto, D., LeRoux, J.A., Wilson-Cohn, C., Raroque J., Kooken K, et al. (2000). A new test to measure emotion recognition ability: Matsumoto and Ekman's Japanese and Caucasian Brief Affect Recognition Test
(JACBART). Journal of Nonverbal Behaviour, 24, 179-209.

Matthews, G., Zeidner, M., \& Roberts, R. (2012). Emotional Intelligence, 101. New York: Springer Publishing Company.

Maul, A. (2012). The validity of the MayerSalovey-Caruso Emotional Intelligence Test (MSCEIT) as a measure of emotional intelligence. Emotion Review, 4(4), 394-402. doi:10.1177/1754073912445811

Mayer, J. D., Roberts, R. D., \& Barsade, S. G. (2008). Human abilities: Emotional intelligence. Annual Review of Psychology, 59(1), 507-536.

doi:10.1146/annurev.psych.59.103006.093646

Mayer, J. D., Caruso, D., \& Salovey, P. (1999). Emotional intelligence meets traditional standards for an intelligence. Intelligence, 27, 267-298. doi:10.1016/s0160-2896(99)00016-1

Mayer J. D., Caruso D. R., \& Salovey P. (2016). The Ability Model of Emotional Intelligence: Principles and updates. Emotion Review, 8(4), 290-300. doi:10.1177/1754073916639667

Mayer, J. D., \& Salovey, P. (1997). What is emotional intelligence? En P. Salovery y D. Sluyter (Eds.), Emotional development and emotional intelligence: Implications for educators (pp.3-34). New York: Basic Books. (Trad. cast., Pirámide, 2011).

Mayer, J. D., Salovey, P., \& Caruso, D.R. (2002). The Mayer-Salovey-Caruso Emotional Intelligence Test (MSCEIT): User's manual. Toronto: Multi-Health Systems.

Mennin, D., Holaway, R., Fresco, D., Moore, M., \& Heimberg, R. (2007). Delineating components of emotion and its dysregulation in anxiety and mood psycopathology. Behavior Therapy, 38, 284-302. doi:10.1016/j.beth.2006.09.001

Mogg, K., Mathews, A., Bird, C., \& MacGregorMorris, R. (1990). Effects of stress and anxiety on the processing of threat stimuli. Journal of Personality and Social Psychology, 59(6), 1230-1237. doi:10.1037//0022-3514.59.6.1230

Nelis, D., Quoidbach. , Mikolajczak, M., \& Hansenne, M. (2009). Increasing emotional intelligence: (How) is it possible? Personality \& Individual Differences, 47(1), 36-41. doi:10.1016/j.paid.2009.01.046 
Nowicki, S., \& Carton, J. (1993). The measurement of emotional intensity from facial expressions. Journal of Social psychology, 133(5), 749-750.

doi:10.1080/00224545.1993.9713934

O'Sullivan, M. (2007). Trolling for trout, trawling for tuna. The methodological morass in measuring Emotional Intelligence. En G. Matthews, M. Zeidner y R.D. Roberts (Eds.), The science of emotional intelligence. Knows and unknows (pp. 258-287). New York: Oxford University Press.

Pérez-González, J. C. (2010). Trait emotional intelligence operationalized through the TEIQue: Construct validity and psychopedagogical implications. Unpublished $\mathrm{PhD}$ dissertation. Universidad Nacional de Educación a Distancia (UNED).

Pérez-González, J. C., Petrides, K. V., \& Furnham, A. (2007). La medida de la inteligencia emocional rasgo. En J.M. Mestre Navas y P. Fernández Berrocal (Coord.), Manual de Inteligencia Emocional (pp. 8197). Madrid: Pirámide.

Pérez-González, J. C., \& Qualter, P. (2018). Emotional intelligence and emotional education in school years. En L. Dacree Pool y P. Qualter (Eds.), An Introduction to Emotional Intelligence. Chichester, West Sussex: Wiley.

Perez-Sayes, G., Luna, P., \& Tirapu, J. (2009). Versión revisada del Test de "La lectura de la Mente en los Ojos". Ubarmin Clinic, Brain Injury Unit, Pamplona: ARC Tests.

Petrides K. V., Mikolajczak M., Mavroveli S., Sanchez-Ruiz M. J., Furnham A., \& PérezGonzález J. C. (2016). Developments in trait emotional intelligence research. Emotion Review, 8, 1-7. doi:10.1177/1754073916650493

Pitterman, H., \& Nowicki, S. (2004). A test of the ability to identify emotion in human standing and sitting postures: The Diagnostic Analysis of Nonverbal Accuracy-2 Posture Test (DANVA2-POS). Genetic, Social \& General Psychology Monographs, 130(2), 146-162. doi:10.3200/mono.130.2.146-162

Qualter, P., Davis, S. K., Keefer, K. V., Parker, J. D. A., Saklofske, D. H., Wigelsworth, M., Simmons, N., \& Stough, C. (2017) Emotional competency in education: Core concepts and applications. British Journal of Educational Psychology, 12, 51-71.

Rivers, S. E., Brackett, M. A., Salovey, P., \& Mayer, J. D. (2007). Measuring emotional intelligence as a set of mental abilities. En G. Matthews, M. Zeidner y R. D. Roberts (Eds.), The science of emotional intelligence. Knows and unknows (pp. 230-257). New York: Oxford University Press.

Roberts, R. D., Schulze, R., O'Brien, K., MacCann, C., Reid, J., \& Maul, A. (2006). Exploring the validity of the Mayer-SaloveyCaruso Emotional Intelligence Test (MSCEIT) with established emotions measures. Emotion, 6, 663-69. doi:10.1037/1528-3542.6.4.663

Rode, J. C., Mooney, C. H., Arthaud-day, M. L., Near, J. P., Rubin, R. S., Baldwin, T. T., \& Bommer, W. H. (2008). An examination of the structural, discriminant, nomological, and incremental predictive validity of the MSCEIT $\odot$ V2.0. Intelligence, 36, 350-366. doi:10.1016/j.intell.2007.07.002

Rodrigo-Ruiz, D. (2017). Evaluación de la inteligencia emocional capacidad en adolescentes españoles: Adaptación y validación del Situational Test of Emotion Management (STEM) (tesis doctoral). UNED, Madrid.

Rodrigo-Ruiz, D., Pérez-González J. C., \& Cejudo, J. (2017). Dificultades de reconocimiento emocional facial en niños con TDAH. Revista de Neurología, 65, 145-52.

Rosenthal, R., Hall, J. A., DiMatteo, M. R., Rogers, P. L., \& Archer, D. (1979). Sensitivity to nonverbal communications: The PONS Test. Baltimore, MD: The Johns Hopkins University Press.

Scherer, K. R., Banse, R., \& Wallbott, H. G. (2001). Emotion inferences from vocal expression correlate across languages and cultures. Journal of Cross-Cultural Psychology, 32, 76-92. doi:10.1177/0022022101032001009

Scherer, K. R., \& Scherer, U. (2011) Assessing the ability to recognize facial and vocal expressions of emotion: Construction and validation of the Emotion Recognition Index. Journal of Nonverbal Behavior, 35(4), 305326. doi:10.1007/s10919-011-0115-4 
Schulze, R., Wilhelm, O., \& Kyllonen, P. C. (2007). Approaches to the assessment of emotional intelligence. En G. Matthews, M. Zeidner y R.D. Roberts (Eds.), The science of emotional intelligence. Knows and unknows (pp. 202-229). New York: Oxford University Press.

Sharma, S., Gangopadhyay, M., Austin, E., \& Mandal, M. K. (2013). Development and validation of situational judgment test of emotional intelligence. International Journal of Selection \& Assessment, 21(1), 57-73. doi:10.1111/ijsa.12017

Sharma, D., \& McKenna, F. P. (2001). The role of time pressure on the emotional Stroop task. British Journal of Psichology, 92, 471-481. doi:10.1348/000712601162293

Shimokawa, A., Yatomi, N., Anamizu,S., Ashikari, I., Kohno, M., Maki, Y., Torii, S., Isono, H., Sugai, Y, Koyama, N., \& Matsuno, Y. (2000). Comprehension of emotions: Comparison between Alzheimer type and vascular type dementias. Dementia and Geriatric Cognitive Disorders, 11(5), 268274. doi:10.1159/000017249

Siegling, A.B., Saklofske, D.H., y Petrides, K.V. (2015). Measures of ability and trait emotional intelligence. En G.J. Boyle, D.H. Saklofske y G. Matthews (Eds.) Measures of Personality and Social Psychological Constructs. London: Academic Press.

Tottenham, N., Tanaka, J. W., Leon, A. C., McCarry, T., Nurse, M., Hare, T. A., Marcus, D. J., Westerlund, A., Casey, B. J., \& Nelson, C. (2009). The NimStim set of facial expressions: judgments from untrained research participants. Psychiatry Research, 168(3), 242-249. doi:10.1016/j.psychres.2008.05.006

Zeidner, M., Matthews, G., \& Roberts, R. (2009). What we know about emotional intelligence. How it affects learning, work, relationship and our mental health. Mssachusetts: Institute of Technology. 